\title{
Collapsar and Magnetar Models for Long-Duration Gamma-Ray Bursts
}

\author{
Shigehiro Nagataki ${ }^{1}$ \\ Yukawa Institute for Theoretical Physics, Kyoto University \\ Oiwakecho, Kitashirakawa, Sakyo, Kyoto, Japan \\ E-mail: nagataki@yukawa.kyoto-u.ac.jp
}

\begin{abstract}
I have developed 2- and 3-dimensional general relativistic magnetohydrodynamic (GRMHD) code. I performed numerical simulations of collapsars using these codes and realistic progenitor models. In the GRMHD simulation, it was shown that a jet is launched from the central region of the progenitor as long as the central black hole is rotating rapidly. I performed simulations of collapsars with different Kerr parameters $\mathrm{a}=0,0.5,0.9,0.95$. It is shown that a more rapidly rotating black hole is driving a more energetic jet. No jet is seen for the case of Schwartzschild black hole case, while the total energy of the jet is as large as $10^{50} \mathrm{erg}$ for a rapidly rotating Kerr black hole case $(\mathrm{a}=0.95)$. In order to explain the high luminosity of a GRB, it is concluded that a rapidly rotating black hole is favored ('faster is better'). I also started to perform 3-dimensional GRMHD simulation of collapsars.
\end{abstract}

Gamma-Ray Bursts 2012 Conference

Munich, Germany

May 7-11, 2012

$1 \quad$ Speaker 


\section{Introduction}

It is still unknown how the central engine of Long Gamma-Ray Bursts (hereafter it is called as GRBs for simplicity) is working at the center of massive stars. In other words, we poorly know the engine that drives the most powerful explosion in the universe. Some of the supernovae that associate with GRBs were very energetic. Their energies were of the order of $10^{52} \mathrm{ergs}$, which is about 10 times larger than the ones of normal core-collapse supernovae. This fact strongly suggests that a different engine from the one of normal core-collapse supernovae should be working at the center of the GRB progenitors.

There are some possible scenarios to drive a GRB jet. One of the most promising scenarios is the collapsar scenario. In this study, we investigate the collapsar scenario. In the collapsar scenario, a black hole is formed as a result of gravitational collapse. Also, rotation of the progenitor plays an important role. Due to the rotation, an accretion disk is formed around the equatorial plane. After the formation of the accretion disk, there are several possible stories as below.

One is that a jet-induced explosion along the rotation axis may occur due to the heating through neutrino anti-neutrino pair annihilation that are emitted from the accretion disk. This is an interesting possibility, although neutrino heating efficiency looks small [1]. A very careful treatment of neutrino transfer with conserved-scheme of hydrodynamics will be necessary to prove that this effect is the key-process of the GRB engine.

Another is that a jet is driven by extracting rotational energies of the accretion disk with a help of magnetic fields that pierce the disk (Blandford-Payne effect). This scenario is also investigated by several authors. This is also a promising possibility. Further study will be necessary whether a relativistic jet will be launched by this mechanism.

Recently, the effect of extraction of rotation energy from the black hole through outgoing Poynting flux (Blandford-Znajek effect) is investigated by using a General Relativistic Magneto-hydro Dynamics (GRMHD) code. Energy extraction from a rotating black hole is a general relativistic effect, so a GRMHD code is necessary to investigate the effect. In [2], they showed that a jet is launched by the Blandford-Znajek effect using a Kerr black hole with Kerr parameter $(\mathrm{a}=0.9)$ and a polytrope density profile for a massive star model. In their successive papers [3]-[4], they also succeeded to launch a jet by Blandford-Znajek effect (Kerr Parameter was chosen to be 0.9 in [3], while 0.46 and 0.6 in [4]) using a polytrope density profile for a massive star model. In [5], it was shown that a jet is launched by the Blandford-Znajek effect (Kerr parameter was chosen to be 0.5) using a realistic progenitor model. In [5], it was pointed out that the Blandford-Payne effect may be also working.

As stated above, in the previous papers, it was shown that a jet is successfully launched by the Blandford-Znajek effect. However, there is no systematic study how the dynamics depends 
on the Kerr parameter. It is true that [4] presented results for different Kerr parameters, but they also changed the amplitude of the initial magnetic fields. Also, no simulation has been reported for $\mathrm{a}=0$, that is, the Schwartzschild black hole case. By performing a simulation for this case, we can clearly see how effectively the rotating black hole is working to drive the jet. In this paper, I present 4 simulations for the same initial condition with [5], but with different Kerr parameters $\mathrm{a}=0,0.5,0.9,0.95$. It is shown clearly that a more rapidly rotating black hole is driving a more energetic jet. Especially, in the case of the Schwartzschild case, no jet is found. That proves that the jet is really driven by the rotating black hole [6]. Also, recently I started to perform 3dimensional GRMHD simulation of collapsars. In this paper, I would like to show my preliminary result on that [7].

\section{Method of Calculation}

In [5], I have developed a two-dimensional GRMHD code following [8]. I have adopted a conservative, shock-capturing scheme with Harten, Lax, and van Leer (HLL) flux term and flux-interpolated constrained transport technique. I use a third-order Total Variation Diminishing (TVD)

Runge-Kutta method for evolution in time, while a monotonized central slope-limited linear interpolation method is used for second-order accuracy in space. A 2D scheme (2dimensional Newton-Raphson method) is usually adopted for transforming conserved variables to primitive variables.

When I perform simulations of GRMHD, the Modified Kerr-Schild coordinate is basically adopted with mass of the black hole (M) fixed where the Kerr-Schild radius ( $r$ ) is replaced by the logarithmic radial coordinate $\mathrm{x}_{1}=\ln \mathrm{r}$. When I show the result, the coordinates are transfered from the Modified Kerr-Schild coordinates to Kerr-Schild ones.

In the following, I use $\mathrm{G}=\mathrm{M}=\mathrm{c}=1$ unit. $\mathrm{G}$ is the gravitational constant, $\mathrm{c}$ is the speed of light, and $\mathrm{M}$ is the gravitational mass of the black hole at the center.

The calculated region corresponds to a quarter of the meridian plane under the assumption of axisymmetry and equatorial symmetry. The spherical mesh with $256(\mathrm{r})$ by $128(\theta)$ grid points is used for all the computations. The radial grid is non-uniform, extending from $r=0.98 r_{+}$to $3 \times 10^{4}$ ( $\mathrm{r}_{+}$is the outer horizon, and $3 \times 10^{4}$ corresponds to $8.9 \times 10^{9} \mathrm{~cm}$ in cgs units) with uniform grids in the Modified Kerr-Schild coordinate.

I adopt the model 12TJ in [9]. I assume that the central part of the star has collapsed and formed a black hole of 2 times the solar mass. In this case, $\mathrm{r}=1$ corresponds to $2.95 \times 10^{5} \mathrm{~cm}$. I also assume that the gravitational mass of the black hole is unchanged throughout the calculation. In [5], the Kerr parameter, a, was assumed to be 0.5, while in [6] I performed simulations for $\mathrm{a}=0,0.5,0.9,0.95$, respectively. 
I assume the weak vector potential $\mathrm{A}_{\varphi}$ and the poloidal field is normalized so that the minimum value of $\mathrm{p}_{\mathrm{gas}} / \mathrm{p}_{\mathrm{mag}}=10^{2}$ where $\mathrm{p}_{\mathrm{gas}}$ is the thermal pressure and $\mathrm{p}_{\mathrm{mag}}$ is the magnetic pressure. I use a simple equation of state $p_{\text {gas }}=(\Gamma-1) \mathrm{u}$ where $\mathrm{u}$ is the internal energy density. $\Gamma=4 / 3$ was chosen so that the equation of state roughly represents radiation gas.

As for the boundary condition in the radial direction, I adopt the outflow boundary condition for the inner and outer boundaries. As for the boundary condition in the zenith angle direction, the axis of symmetry boundary condition is adopted for the rotation axis, while the reflecting boundary condition is adopted for the equatorial plane. As for the magnetic fields, the equatorial symmetry boundary condition, in which the normal component is continuous and the tangential component is reflected, is adopted.

As for 3-dimensional calculations, I used the same progenitor model with the spherical mesh with $256(\mathrm{r})$ by $256(\theta)$ by $32(\phi)$ grid points. As for the theta direction, the whole space is covered in the simulation $(0<\theta<\pi)[7]$.

\section{Results}

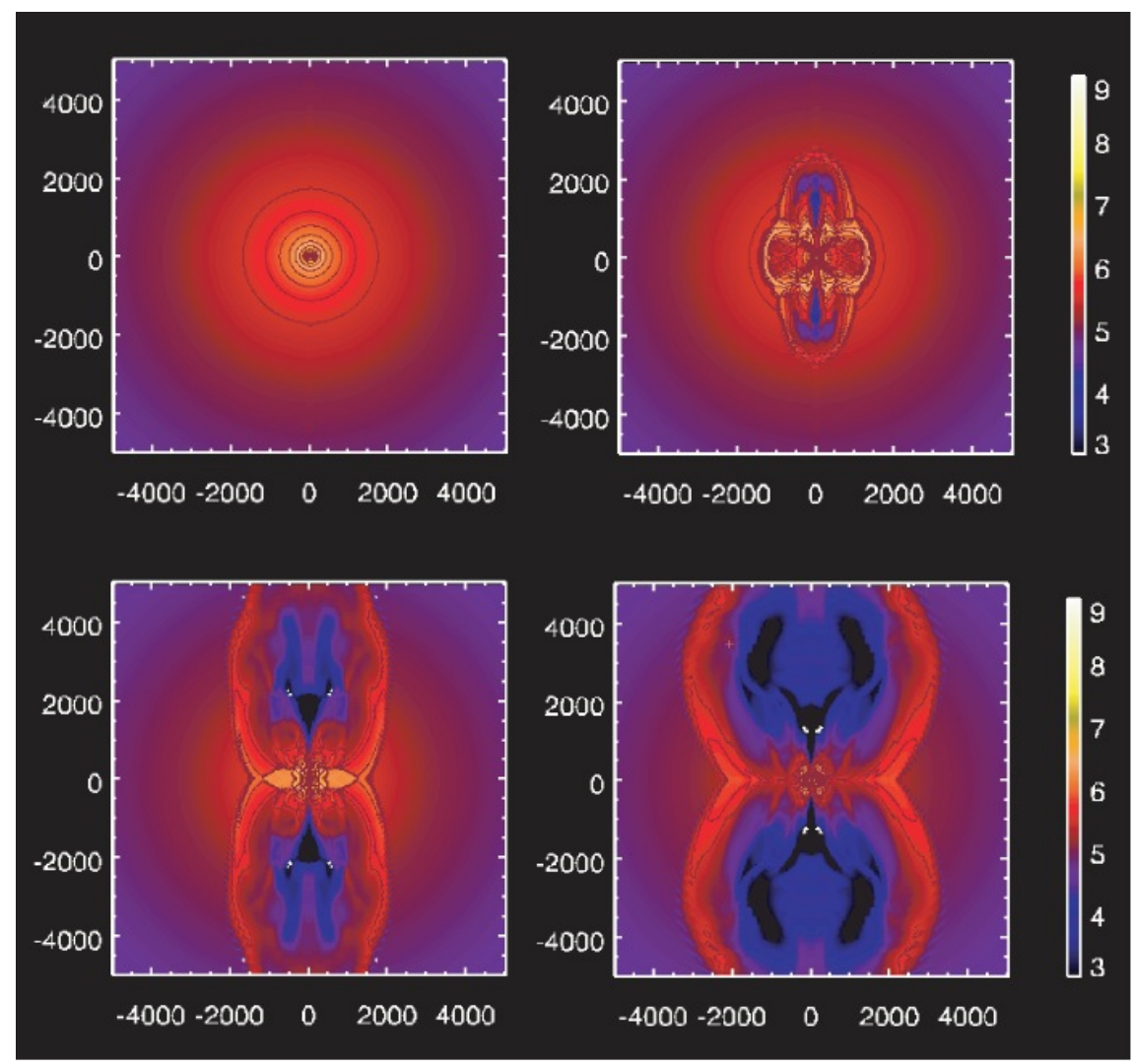

Figure 1. Contours of rest mass density in logarithmic scale for all models at the same time-slice $1.5760 \mathrm{sec}$.

In figure 1, contours of rest mass density in logarithmic scale are shown for all models at the same time-slice $1.5760 \mathrm{sec}$. Cgs units are used for the rest mass density, while the length in 
the vertical/horizontal axes is written in $\mathrm{G}=\mathrm{M}=\mathrm{c}=1$ unit. $\mathrm{r}=1$ and 4000 corresponds to $2.95 \times 10^{5}$ $\mathrm{cm}$ and $1.18 \times 10^{9} \mathrm{~cm}$, respectively. These results are projected on the $(\mathrm{r} \sin (\theta), \mathrm{r} \cos (\theta))$-plane. Upper left panel shows the state of $a=0$, upper right panel shows the one of $a=0.5$, lower left panel shows the one of $a=0.9$, and lower right panel shows the one of $a=0.95$. It is clearly shown that the rotating black hole drives the jet (the Schwartzschild black hole cannot drive a jet, while a more rapidly rotating black hole is driving a stronger jet).

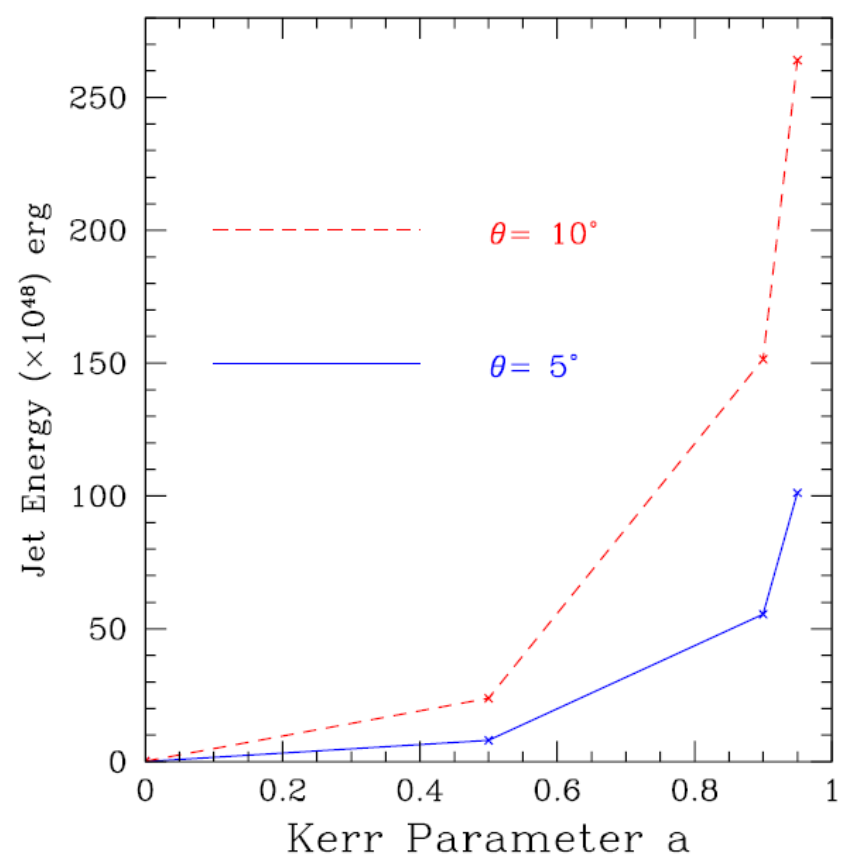

Figure 2. Plots of the jet energy for all models $(a=0,0.5,0.9,0.95)$

In figure 2, plots of the jet energy for all models at $t=160000$ are shown. It is noted that the contribution of the rest mass energy is subtracted. The blue curve represents the jet energy within the opening angle theta $=5$ degree, while the red curve represents the one within theta $=$ 10 degree. The unit of vertical axis is $10^{48} \mathrm{erg}$. It is clearly seen that a more rapidly rotating black hole is driving a stronger jet. The total energy of the jet for $\mathrm{a}=0.95$ is as large as $10^{50} \mathrm{erg}$. Thus it is concluded that 'faster is better' (rapidly rotating black hole is better to drive a GRB jet).

I am extending my study on collapsars by performing 3-dimensional simulations. In figure 3, an example of the 3-dimensional simulation is shown. The progenitor model is same with the 2-D simulations, and Kerr parameter is chosen to be 0.9 in this simulation. The basic picture of the dynamics is same with 2-D. Further investigation will be presented in the near future. 


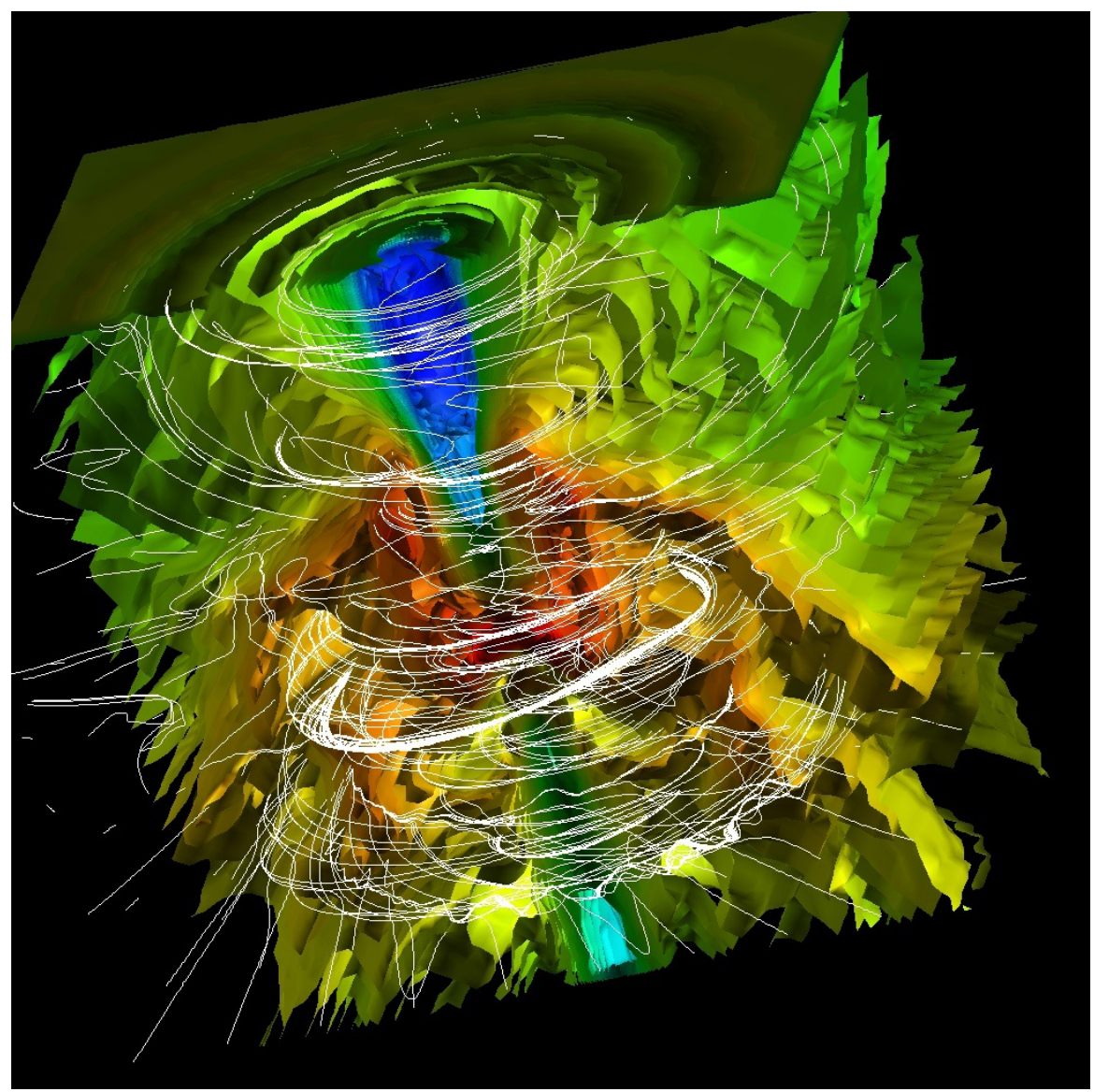

Figure 3. Density contour with B-Field curves of 3-dimensional GRMHD simulation.

\section{Photospheric emission model}

We are studying not only the central engine of long-GRBs but also other aspects of GRBs such as explosive nucleosynthesis [10]-[11], propagation of relativistic jets [12]-[13], UHECR-VHE neutrino production in GRBs [14]-[16], and supernova remnant phase [17]. In this paper, I would like to show our recent result on the photospheric emission model [18].

We have explored the photospheric emissions from ultra-relativistic jets which has a spine-sheath structure. It is assumed that the fast spine jet is embedded in a slower sheath outflow. We solved the propagation of thermal photons which are injected in regions of high optical depth. Due to the presence of shear velocity in the boundary of the spine and sheath region, fraction of the injected photons are accelerated and the form a high energy power-law tail in the resultant spectrum. We show, in particular, that the result can successufuly reproduce the spectra of observed prompt emissions of gamma-ray bursts. 
An example of the resulting spectrum in the observer's frame is shown in the right panel of figure 4. The parameters assumed in this example are shown in the figure. It is noted that the Band-like spectrum is realized in the example. This may shed light on the understanding of SED of GRBs [18].
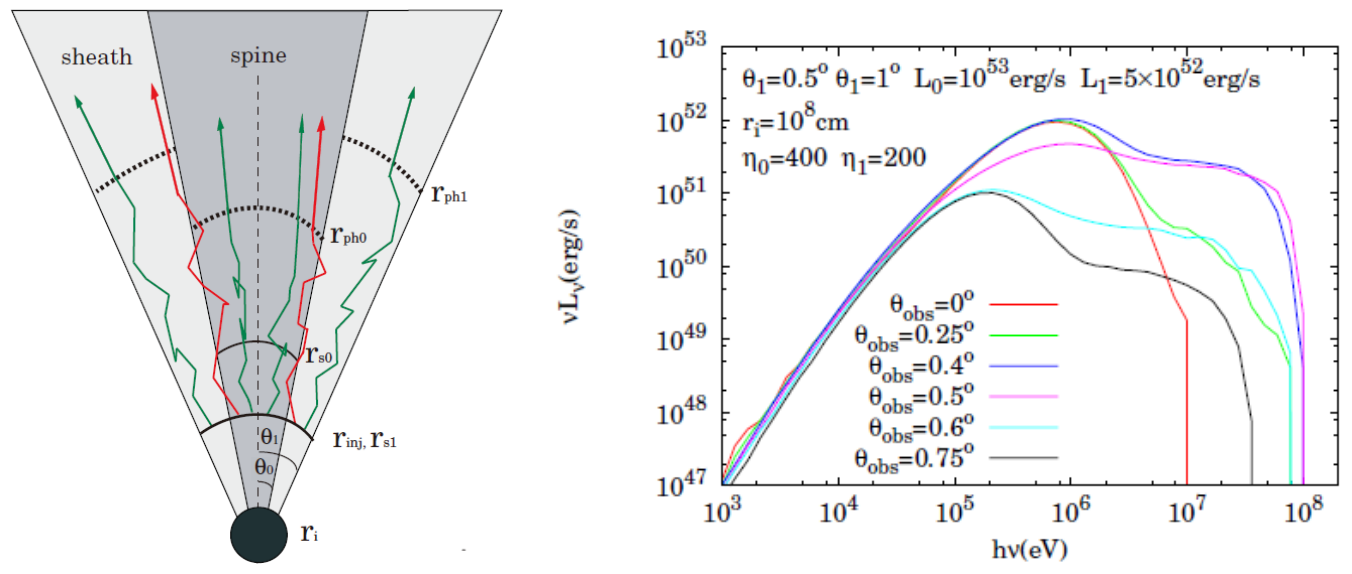

Figure 4. Left: Schematic picture of the spine-sheath structure and propagation of gammarays in the flow. Right: An example of resulting SED.

\section{References}

[1] S. Nagataki, R. Takahashi, A. Mizuta, T. Takiwaki, Numerical Study on GRB-Jet Formation in Collapsars, ApJ 659 (2007) 512-529.

[2] M.V.Barkov and S.S. Komissarov, Stellar explosions powered by the Blandford-Znajek mechanism, MNRAS 385 (2008) L28

[3] S.S. Komissarov and M.V. Barkov, Activation of the Blandford-Znajek mechanism in collapsing stars, MNRAS 397 (2009) 1153

[4] M.V. Barkov and S.S. Komissarov, Close binary progenitors of gamma-ray bursts, MNRAS 401 (2010) 1644

[5] S. Nagataki, Development of General Relativistic Magnetohydrodynamic Code and its Application to Central Engine of Long Gamma-Ray Bursts, ApJ 704 (2009) 937

[6] S. Nagataki, Rotating Black Holes as Central Engines of Gamma-Ray Bursts: Faster is Better, PASJ 63 (2011) 1243

[7] S. Nagataki 2012, in preparation

[8] C.F. Gammie, J. McKinney, G. Toth, HARM: A Numerical Scheme for General Relativistic Magnetohydrodynamics, ApJ $\mathbf{5 8 9}$ (2003) 444

[9] S.E.Woosley and A.Heger, The Progenitor Stars of Gamma-Ray Bursts, ApJ 637 (2006) 914

[10]S. Nagataki, A.Mizuta, S.Yamada, H.Takabe, K.Sato, Explosive Nucleosynthesis Accompanied 
with Formation of Jet-induced GRBs in Massive Stars. ApJ 596 (2003) 401

[11] S. Nagataki, A. Mizuta, K. Sato, Explosive Nucleosynthesis in GRB Jets Accompanied by Hypernovae, ApJ 647 (2006) 1255

[12] A. Mizuta, T. Yamasaki, S. Nagataki, S. Mineshige, Collimated Jet or Expanding Outflow: Possible Origins of GRBs and X-ray Flashes, ApJ 651 (2006) 960

[13] A. Mizuta, S. Nagataki, J. Aoi, Thermal Radiation from GRB Jets, ApJ 732 (2011) 26

[14] K. Murase and S. Nagataki, High energy neutrino emission and neutrino background from gamma-ray bursts in the internal shock model, PRD 73 (2006) 063002

[15] K. Murase and S. Nagataki, High Energy Neutrino Flash from Far-UV/X-ray Flares of Gamma-Ray Bursts, PRL 97 (2006) 051101

[16] H. He, R.-Y. Liu, X.-Y. Wang, S. Nagataki, K. Murase, Z.-G. Dai, IceCube non-detection of GRBs: Constraints on the Fireball Composition and Implications for UHECRs, ApJ 752 (2012) 29

[17] S.-H. Lee, D. Ellison, S. Nagataki, A Generalized Model of Nonlinear Diffusive Shock Acceleration Coupled to an Evolving Supernova Remnant, ApJ 750 (2012) 156

[18] H.Ito,S. Nagataki, et al. 2012, in prep 